\title{
Pre- or post-treatment with ethanol and ethyl pyruvate results in distinct anti-inflammatory responses of human lung epithelial cells triggered by interleukin-6
}

\author{
BORNA RELJA ${ }^{1}$, NINA OMID ${ }^{1}$, ALEXANDER SCHAIBLE ${ }^{1}$, MARIO PERL ${ }^{2}$, \\ SIMON MEIER $^{1}$, ELSIE OPPERMANN $^{3}$, MARK LEHNERT $^{1}$ and INGO MARZI ${ }^{1}$
}

\author{
${ }^{1}$ Department of Trauma, Hand and Reconstructive Surgery, University Hospital, Goethe University, Frankfurt am Main D-60590; \\ ${ }^{2}$ Department of Trauma Surgery, Trauma Center Murnau, Murnau D-82418; ${ }^{3}$ Department of General Surgery, \\ University Hospital, Goethe University, Frankfurt am Main D-60590, Germany
}

Received May 22, 2014; Accepted February 5, 2015

DOI: $10.3892 / \mathrm{mmr} .2015 .3764$

\begin{abstract}
Increased local and systemic levels of interleukin (IL)-6 are associated with inflammatory processes, including neutrophil infiltration of the alveolar space, resulting in lung injury. Our previous study demonstrated the beneficial anti-inflammatory effects of acute exposure to ethanol (EtOH) in an acute in vivo model of inflammation. However, due to its side-effects, EtOH is not used clinically. In the present study, the effects of EtOH and ethyl pyruvate (EtP) as an alternative anti-inflammatory drug prior to and following application of an IL-6 stimulus on cultured A549 lung epithelial cells were compared, and it was hypothesized that treatment with $\mathrm{EtOH}$ and EtP reduces the inflammatory potential of the A549 cells. Time- and dose-dependent release of IL-8 from the A549 cells was observed following stimulation with IL-6. The release of IL-8 from the A549 cells was assessed following treatment with EtP (2.5-10 mM), sodium pyruvate (NaP; $10 \mathrm{mM})$ or EtOH (85-170 mM) for 1, 24 or $72 \mathrm{~h}$, prior to and following IL-6 stimulation. The adhesion capacities of neutrophils to the treated A549 cells, and the expression levels of cluster of differentiation (CD)54 by the epithelial cells were measured. Treatment of the A549 cells with either EtOH or EtP significantly reduced the IL-6-induced release of IL-8. This effect was observed in the pre- and post-stimulatory conditions, which is of therapeutic importance. Similar data was revealed regarding the IL-6-induced neutrophil adhesion to the treated A549 cells, in which pre- and post-treatment with EtOH or EtP decreased the adhesion capacity, however, the results were dependent on the duration of incubation. Incubation durations of 1 and $24 \mathrm{~h}$ decreased the adhesion rates of neutrophils to
\end{abstract}

Correspondence to: Dr Borna Relja, Department of Trauma, Hand and Reconstructive Surgery, University Hospital, Goethe University, Theodor-Stern-Kai 7, Frankfurt am Main D-60590, Germany E-mail: info@bornarelja.com

Key words: ethanol, ethyl pyruvate, lung, inflammation, in vitro the stimulated A549 cells, however, the reduction was only significant at $72 \mathrm{~h}$ post-treatment. The expression of CD54 was reduced only following treatment for $24 \mathrm{~h}$ with either EtOH or EtP, prior to IL-6 stimulation. Therefore, EtOH and EtP reduced the inflammatory response of lung epithelial cells, and the potential of EtP to mimic EtOH was observed in the pre- and post-treatment conditions.

\section{Introduction}

Alcohol (ethanol; EtOH) is a potent immunomodulatory drug with adverse and inconsistent effects on inflammation (1-4). Its pathological mode of action is dose dependent and varies in acute and chronic exposure (1-8). $\mathrm{EtOH}$ consumption is a risk factor in clinical treatment and has been closely associated with several negative clinical outcomes (9-11). Concerning trauma, one of leading causes of mortality, EtOH has been associated with one third of all traumatic injury-associated mortalities $(9,10)$. In addition, $\sim 50 \%$ of all trauma victims enter emergency departments with positive blood EtOH concentrations (BACs). Numerous studies have reported increased risks of infectious complications, including pneumonia, sepsis, organ and multiple organ failure, during the clinical course following trauma, however, others report conflictive findings (11-17). Within this context, it has been demonstrated that chronic, rather than acute, EtOH abuse adversely affects recovery and outcome following trauma $(1,2)$. Heavy EtOH abuse is detrimental to health and is closely associated with an increased release of pro-inflammatory cytokines (5). However, studies investigating acute and/or low-dose moderate EtOH intake have demonstrated potential beneficial anti-inflammatory effects $(6,7)$. Experimental and epidemiological data have confirmed the reduced risk of cardiovascular disease events, including ischemic heart disease or stroke, diabetes and a lower incidence of admission coagulopathy in severe traumatic brain injury patients (18-20). EtOH-induced suppression of the host defense is associated with decreased polymorphonuclear neutrophil (PMN) migration, and reductions in $\mathrm{PMN}$-adherence to endothelial cells and the productive 
capacity of pro-inflammatory interleukin (IL)-8 (3). These effects may result in increased subsequent susceptibility to infections and also indicate the therapeutic potential of EtOH on the host immunity under acute inflammatory conditions. IL-6, a key active protein in the pathogenesis of diseases of the respiratory system, has been found to be reduced following acute $\mathrm{EtOH}$ intake in an in vivo model of acute inflammation induced by hemorrhagic shock with subsequent resuscitation $(4,21)$. However, support for its use in the clinical environment are sparse and highly limited due to its entry into the central nervous system (CNS). Ethyl pyruvate $(\mathrm{EtP})$, derived from pyruvate and ethanol, is a well-tolerated non-toxic compound with similar anti-inflammatory effects to pyruvate, however, with higher stability $(22,23)$. Its application in vivo leads to significant protective effects from lipopolysaccharide (LPS)-induced white matter injury in the developing rat brain (24). EtP exerts therapeutic effects in a number of experimental in vivo models, including hemorrhagic shock, endotoxemia and severe sepsis $(22,25,26)$. In the lungs, EtP protects against ventilation-induced neutrophil infiltration and oxidative stress (27). Due to its stability, the lack of side effects, the wide therapeutic window and no clear signs of intoxication following its use, EtP has received significant attention due to its potential clinical usefulness for the treatment of acute inflammatory conditions. However, important questions regarding the effects of time and dose in EtOH and EtP, and their possible comparability, remain. In the present study, immortalized human alveolar epithelial cells were treated with EtOH or EtP for various durations and at different dosages, prior to and following treatment with the pro-inflammatory cytokine, IL-6. The aim was to determine whether EtP confers similar beneficial effects as EtOH, in a time- and dose-dependent manner, in an in vitro model of pulmonary inflammation.

\section{Materials and methods}

Cell culture. The human A549 lung adenocarcinoma cell line was purchased from Cell Lines Services (Heidelberg, Germany). The cells were cultured at $37^{\circ} \mathrm{C}$ under $5 \% \mathrm{CO}_{2}$ in $9 \mathrm{ml} \mathrm{RPMI-1640} \mathrm{medium} \mathrm{(Seromed,} \mathrm{Berlin,} \mathrm{Germany),}$ supplemented with $10 \%$ heat-inactivated fetal calf serum (FCS; Invitrogen Life Technologies, Darmstadt, Germany), $100 \mathrm{U} / \mathrm{ml}$ penicillin, $100 \mu \mathrm{g} / \mathrm{ml}$ streptomycin (Gibco-BRL, Karlsruhe, Germany) and $20 \mathrm{mM}$ HEPES buffer (Sigma-Aldrich, Steinheim, Germany). The culture medium was replaced every 2-3 days. The cell viability following stimulation with the various substances, as described in "cell stimulation", was assessed by measuring the levels of the lactate dehydrogenase cytoplasmic enzyme, using an LDL Cytotoxicity Detection kit (Roche, Penzberg, Germany), as described below.

The isolation of blood PMNs from healthy individuals was in accordance with the Declaration of Helsinki. The PMNs were isolated by density-gradient centrifugation at $1124 \mathrm{x} \mathrm{g}$ for $30 \mathrm{~min}$ at room temperature using polymorphprep $(1.113 \pm 0.001 \mathrm{~g} / \mathrm{ml}$; Nycomed, Oslo, Norway), according to the manufacturer's instructions and, as previously reported (28). Following isolation, the PMNs were cultured in RPMI-1640 medium, as described above, and their number and viability were determined using a trypan blue (Gibco-BRL) exclusion assay. Only cell cultures with a purity of $>95 \%$ were used in the subseqent experiments.

Cell stimulation. The concentrations of EtOH, EtP, NaP and IL-6 used were based on previous studies and our previous study to improve comparison of data $(3,8,29-32)$. EtOH was used at 85 and $170 \mathrm{mM}\left(0.5-1 \mathrm{vol}^{-1} \%\right.$; 4-7.9 $\left.\mathrm{mg} \mathrm{EtOH} \mathrm{ml}^{-1}\right)$, as described previously (28-30). Similarly, the concentrations of EtP (2.5 and $10 \mathrm{mM})$ and $\mathrm{NaP}(10 \mathrm{mM})$ were selected, according to previous studies $(18,31)$. The cells were stimulated with either $\mathrm{EtOH}, \mathrm{EtP}$ or $\mathrm{NaP}$ for 1, 24 and $72 \mathrm{~h}$ to mimic acute and chronic EtOH exposure.

The time and dose dependency of the secretory capacity of A549 cells was determined by the stimulation of A549 cells with recombinant human IL-6 (10, 50 or $100 \mathrm{ng} / \mathrm{ml}$; R\&D Systems, Wiesbaden, Germany) for 4, 8, 12 and 24 h (33). Based on the secretory capacity of IL-8, IL- 6 was used at a dose of $10 \mathrm{ng} / \mathrm{ml}$, and the A549 cells were stimulated for $24 \mathrm{~h}$ (Fig. 1), either prior to or following treatment with EtOH, EtP or NaP for various durations (33).

Cell viability. The viabilities of the A540 cells were analyzed by measuring the cytoplasmic levels of $\mathrm{LDH}$. In the case of a damaged plasma membrane, cells release LDH into the cell culture supernatant. The activity of LDH in the supernatants collected from cells treated with EtOH, EtP, NaP and IL-6 in a dose and time-dependent manner was determined enzymatically using a cytotoxicity detection kit (LDH) (Roche), according to the manufacturer's instructions. The A549 viability was $>95 \%$ at all the time points and doses selected for the treatment of the cells in these experiments. In addition, no or marginal detachment of the cells was detected by microscopic evaluation of cell layers following treatment with EtP using an Axio Observer.Z1 (Zeiss, Göttingen, Germany).

A trypan blue exclusion assay was performed to determine the level of viability of the PMNs. Briefly, the isolated PMNs were stained with $0.4 \%$ trypan blue and $\sim 100$ cells were counted for each isolation. The mean percentage of viability was $>99 \%$.

Quantification of cytokine production. The A549 cells were incubated with different concentrations of IL-6 over a time course. At each time point, the culture supernatants were harvested and the concentrations of IL-8 were determined using Quantikine assays ( $\& \& D$ Systems), according to the manufacturer's instructions. ELISA was performed using an Infinite M200 microplate reader (Tecan, Männedorf, Switzerland).

To determine the effects of EtOH, EtP and $\mathrm{NaP}$ on the release of IL-8, A549 cells pre- or post-incubation with EtOH, EtP or NaP for 1,24 or $72 \mathrm{~h}$ prior to or following stimulation with IL-6 for $24 \mathrm{~h}$. The expression levels of IL-8 were subsequently measured in the culture supernatants, as described above. To determine differences in the release of cytokines between the different treatments, cytokine levels are expressed as a percentage relative to that of the stimulated controls.

Monolayer adhesion assay. Following pre or post-incubation with $\mathrm{EtOH}, \mathrm{EtP}$ or $\mathrm{NaP}$ for 1, 24 or $72 \mathrm{~h}$ and stimulation with 
IL- 6 for $24 \mathrm{~h}$, the adhesion of PMNs to the treated A549 cells was evaluated. To analyze this, the A549 cells were transferred into 24-well multiplates (Falcon Primaria; Becton Dickinson, Heidelberg, Germany) in complete RPMI-1640 medium (500 $\mu 1$ ). The treatment was performed, as described above, at a confluency of $\sim 80 \%$. Freshly isolated PMNs $\left(5 \times 10^{4}\right.$ cells/well) were carefully added to the A549 monolayer or to an empty plastic surface for $60 \mathrm{~min}$. Subsequently, non-adherent PMNs were washed off three times using warmed $\left(37^{\circ} \mathrm{C}\right)$ complete RPMI-1640 medium. The remaining PMNs were fixed with $1 \%$ glutaraldehyde (Carl Roth, Kalsruhe, Germany). The adherent PMNs were counted in five randomly selected fields of a defined size $\left(5 \times 0.25 \mathrm{~mm}^{2}\right)$ using a phase contrast microscope (20X objective; Axio Observer.Z1) and the mean cellular adhesion rate was calculated.

CD54 surface expression. Following pre- or post-treatment with EtOH, EtP and NaP, and stimulation with IL-6, the A549 cells were washed in phosphate-buffered saline (PBS; Invitrogen Life Technologies) containing $0.5 \%$ bovine serum albumin (BSA; Sigma-Aldrich), and were subsequently incubated with a fluorescein-conjugated mouse monoclonal antibody directed against intercellular adhesion molecule (ICAM)-1/cluster of differentiation (CD)54 (1:20; cat. no. BBA20; clone BBIG-I1; R\&D Systems) for $60 \mathrm{~min}$ at $4^{\circ} \mathrm{C}$. A mouse immunoglobulin G1 fluorescein antibody (1:20; cat. no. IC002F; clone 11711; R\&D Systems) was used as an isotype control. After staining the cells were washed with ice cold PBS (supplemented with $0.5 \%$ BSA), and centrifuged at $259 \mathrm{x} \mathrm{g}$ for $5 \mathrm{~min}$ at $4^{\circ} \mathrm{C}$ to remove any unbound antibody. Subsequently, the PBS was removed and the cells were resuspended in $500 \mu 1$ ice cold PBS (supplemented with $0.5 \%$ BSA). The expression of CD54 was measured by flow cytometry using a FACSCalibur (BD Biosciences, Heidelberg, Germany; $1 \times 10^{4}$ cells/scan), with data expressed as the mean fluorescence units.

Statistical analysis. All experiments were performed between three and six times, and differences between the groups were determined using Wilcoxon-Mann-Whitney U-test. $\mathrm{P}<0.05$ was considered to indicate a statistically significant difference. The data are expressed as the mean \pm standard error of the mean. All statistical analyses were performed using GraphPad Prism 5 software (Graphpad Software, Inc., San Diego, CA).

\section{Results}

IL-8 release from the A549 cells. To determine the secretory potential and the time- and dose- responses of the A549 cells to the IL- 6 pro-inflammatory mediator, the release of IL- 8 by the A549 cells following IL-6 stimulation was evaluated.

IL-6 induced the dose- and time-dependent release of IL-8 (Fig. 1). Treatment with IL-6 at 10,50 and $100 \mathrm{ng} / \mathrm{ml}$ increased the release of IL- 8 continuously as the dose and the duration of incubation increased (Fig. 1). The dose response curve at all three doses peaked at 12/24 h. Therefore, the lowest dose of IL-6 (10 ng/ml) was used for subsequent experiments.

IL-8 release following treatment with EtOH or EtP. It has been reported that a short duration of incubation with EtP

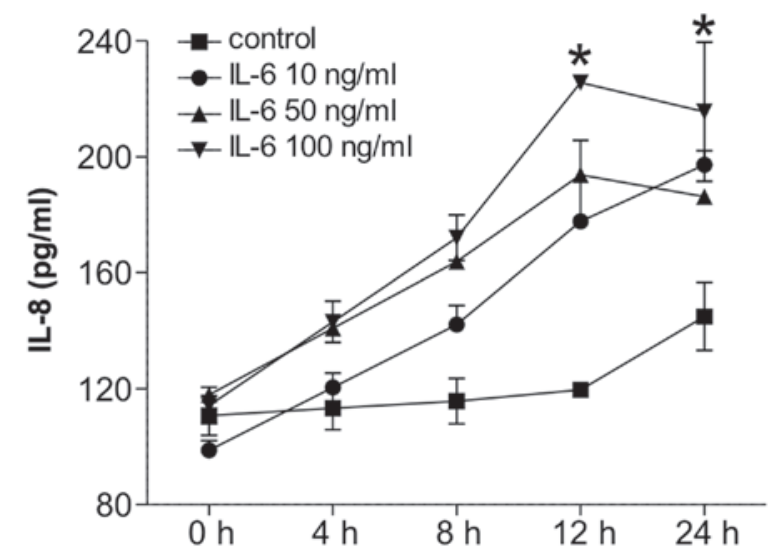

Figure 1. Release of IL-8 from A549 lung epithelial cells following IL-6 stimulation. The cells were stimulated with IL- 6 at the indicated concentrations for different durations. Following the incubation periods, the supernatants were analyzed for IL- 8 concentrations. The data are expressed as the mean \pm standard error of the mean. ${ }^{*} \mathrm{P}<0.05$, all IL- 6 groups, vs. corresponding control. IL, interleukin.

reduces the release of IL-8 in stimulated human endothelial and epithelial cells $(3,29)$. The present study assessed the effects of pre- and post-treatment with $\mathrm{EtOH}$, EtP or NaP for 1, 24 and $72 \mathrm{~h}$ on the IL-6-induced secretory potential of A549 cells to produce IL-8.

In the A549 cells, IL-6 induced a significant increase in the release of IL-8, as shown in Figs. 1 and 2. Pre-treatment with $\mathrm{EtOH}$ for $1 \mathrm{~h}$ (Fig.2) had no effect on the release of IL-8, whereas pre-treatment with EtOH for 24 and $72 \mathrm{~h}$ significantly decreased the release of IL-8 at low $(85 \mathrm{mM})$ and high doses $(170 \mathrm{mM})$ of EtOH, compared with the untreated IL-6-stimulated samples $(\mathrm{P}<0.05)$. Post-treatment with a high dose $\mathrm{EtOH}$ for $1 \mathrm{~h}$ markedly reduced the release of IL-8 from the IL-6-stimulated A549 cells compared with the untreated IL-6-stimulated control cells $(\mathrm{P}<0.05)$. Following post-treatment with $\mathrm{EtOH}$ for 24 or $72 \mathrm{~h}$ at each dose, the release of IL-8 was reduced to levels comparable with those observed pre-treatment $(\mathrm{P}<0.05$; Fig. 2).

Pre-treatment with EtP for $1 \mathrm{~h}$ at each dose had no effect on the release of IL-8, whereas pre-treatment with EtP for 24 or $72 \mathrm{~h}$ significantly decreased the release of IL- 8 at low and high concentrations compared with the untreated IL-6-stimulated samples $(\mathrm{P}<0.05$; Fig. 2). Post-treatment with high concentrations of EtP for $1 \mathrm{~h}$ caused a significant reduction in the IL-6-induced release of IL-8 compared with the untreated control cells $(\mathrm{P}<0.05)$. Following IL-6-stimulation, treatment with low and high doses of EtP for 24 or $72 \mathrm{~h}$ conferred significant decreases in the release of IL-8 (Fig. 2). Treatment with $\mathrm{NaP}$ exerted similar effects to those observed following treatment with high doses of EtP, independently of the pre- or post-treatment condition (Fig. 2).

PMN adherence. The adhesion rates of PMN to the A549 monolayer were significantly increased following IL-6 stimulation of the A549 cells ( $\mathrm{P}<0.05$; Fig. 3). Pre-treatment of the A549 monolayers with 85 or $170 \mathrm{mM}$ EtOH reduced PMN adhesion only when applied at the high dose for $1 \mathrm{~h}$, compared with the untreated IL-6-stimulated A549 cells $(\mathrm{P}<0.05)$, whereas post-treatment of the A549 monolayers with EtOH signifi- 
A

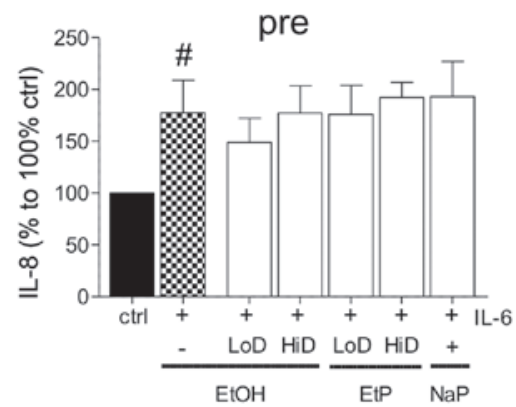

C

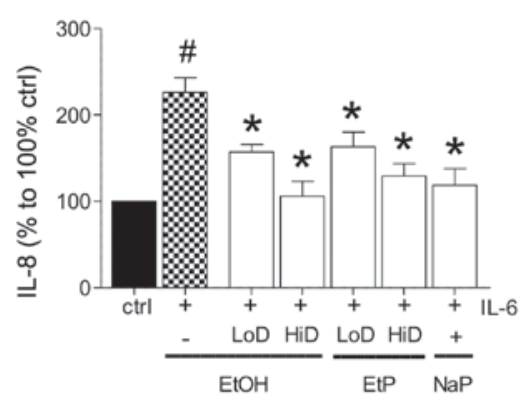

E

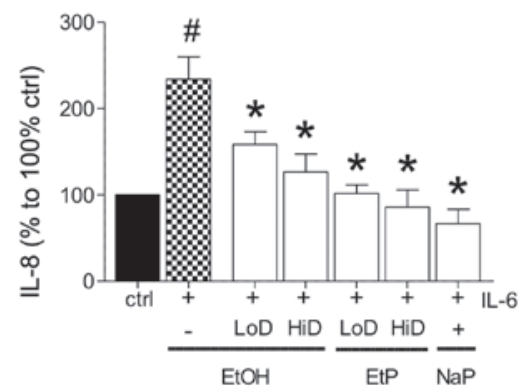

B

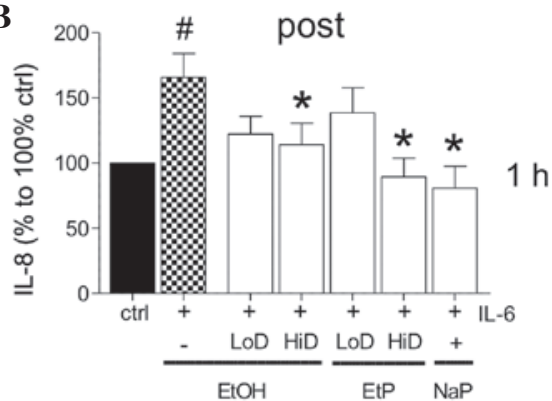

D

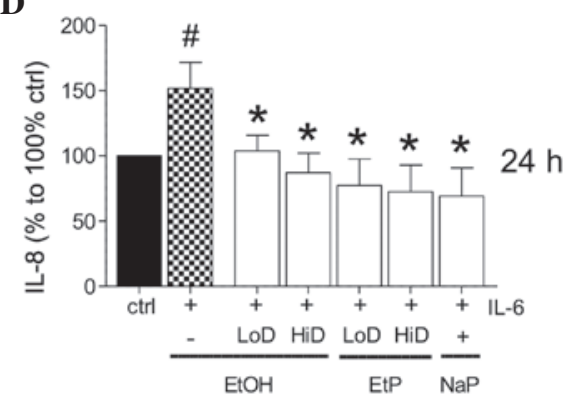

F

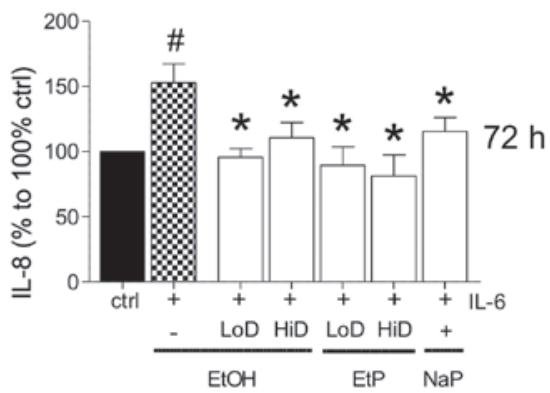

Figure 2. Effects of EtOH, EtP or NaP on the release of IL-8 from A549 lung epithelial cells prior to or following IL-6 stimulation (10 ng/ml for $24 \mathrm{~h}$ ). The cells were treated with EtOH ( $\mathrm{LoD}=85 \mathrm{mM} ; \mathrm{HiD}=170 \mathrm{mM})$, EtP $(\mathrm{LoD}=2.5 \mathrm{mM}, \mathrm{HiD}=10 \mathrm{mM})$ or NaP $(10 \mathrm{mM})$ for $1 \mathrm{~h}(\mathrm{~A})$ pre- and (B) post-IL-6 stimulation; $24 \mathrm{~h}$ (C) pre- and (D) post-IL-6 stimulation; and $72 \mathrm{~h}$ (E) pre- and (F) post-IL-6 stimulation. Following incubation, the supernatants were analyzed for IL-8 concentration and expressed as the percentage of ctrl cells (stimulated with agonists only). The data are expressed as the mean \pm standard error of the mean. ${ }^{*} \mathrm{P}<0.05$, vs. stimulated ctrl; ${ }^{*} \mathrm{P}<0.05$, vs. untreated and unstimulated ctrl. EtOH, ethanol; EtP, ethyl pyruvate; NaP, sodium pyruvate; IL, interleukin; LoD, low dose; HiD, high dose; ctrl, control.

cantly reduced PMN adhesion, compared with the untreated IL-6-stimulated A549 cells independently of incubation duration, $(\mathrm{P}<0.05)$. The only exception was $72 \mathrm{~h}$ post-treatment with a low dose of $\mathrm{EtOH}$, which did not cause a significant reduction in PMN adhesion compared with the control cells (Fig. 3).

Pre-treatment with EtP for $1 \mathrm{~h}$ reduced PMN adhesion only at high concentrations (Fig. 3). Following pre-treatment for $24 \mathrm{~h}$ prior to IL-6 stimulation, EtP reduced the PMN adhesion rates significantly at all doses compared with the untreated IL-6-stimulated control cells $(\mathrm{P}<0.05)$. Pre-treatment for $72 \mathrm{~h}$ at each concentration of EtP did not significantly decrease the PMN adhesion rate. Post-treatment with either low or high doses of EtP for 1, 24 or $72 \mathrm{~h}$ caused a significant reduction in IL-6-induced PMN adhesion rates compared with the untreated IL-6-stimulated control cells $(\mathrm{P}<0.05)$. Treatment with $\mathrm{NaP}$ exerted a significant reduction in PMN adhesion rates only when applied post-treatment, and was independent of the incubation duration (Fig. 3).

Expression of the CD54 adhesion protein. The stimulation of A549 cells with IL-6 caused no significant change in the surface expression of CD54 compared with the unstimulated control cells (Fig. 4). However, the expression of CD54 was significantly reduced following pre-treatment for $24 \mathrm{~h}$ with $\mathrm{EtOH}$, EtP or NaP, independent of the dose used, compared with the control cells $(\mathrm{P}<0.05)$.

\section{Discussion}

Although EtOH intoxication may result in cellular injury and negative clinical outcomes, acute EtOH consumption has several beneficial anti-inflammatory and immunomodulatory effects (4,9-11). Although chronic alcoholism is detrimental to health, acute EtOH consumption may result in a decreased risk of cardiovascular disease events, including stroke, and increased survival rates following significant blood loss in an in vivo model of acute inflammation $(4,18,20)$. However, the use of EtOH clinically is unrealistic due to its well known side-effects to the CNS. The present study evaluated the effects of acute and prolonged EtOH exposure, and the effects of EtP on the inflammatory response of human A549 lung epithelial cells. The A549 cells were exposed to either EtOH 
A

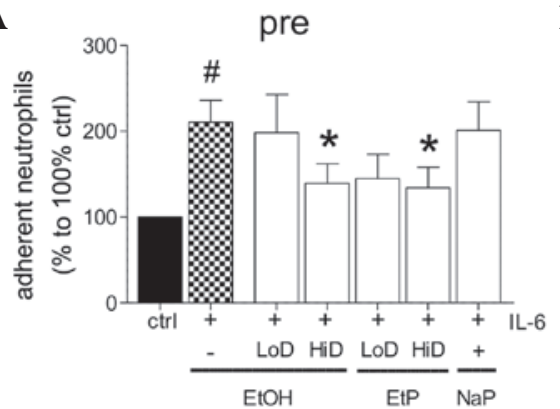

C

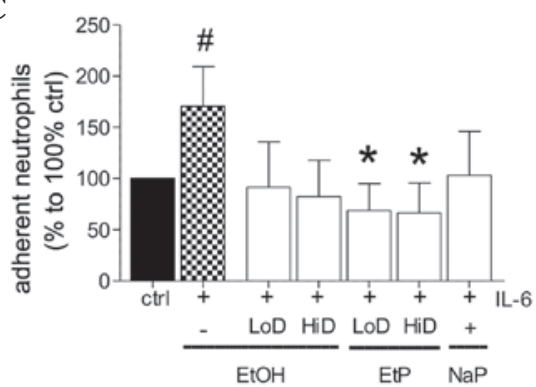

E

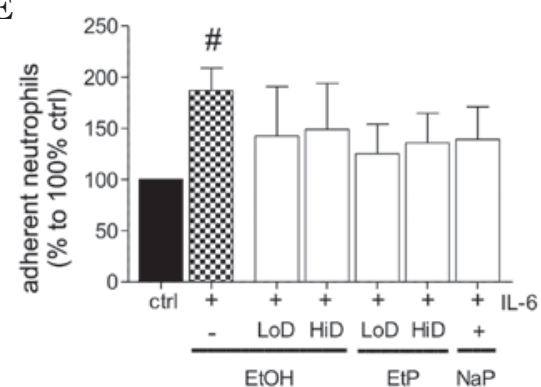

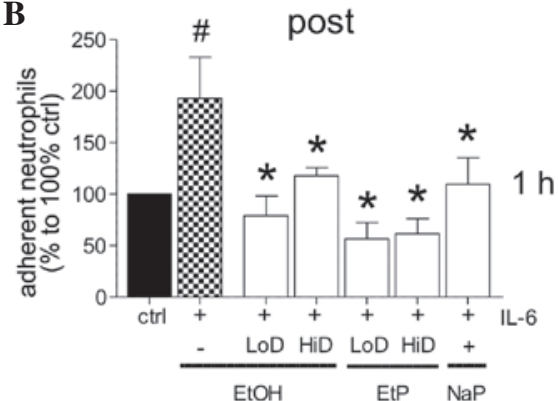

D

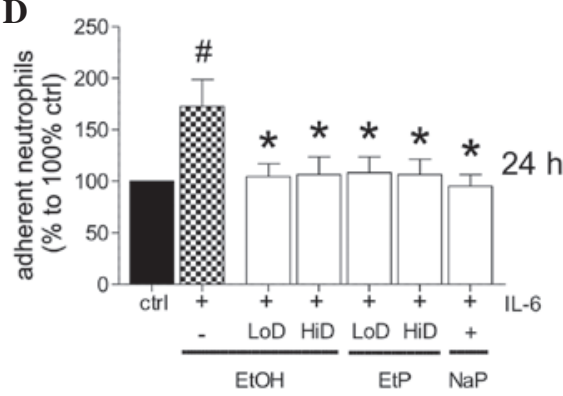

F

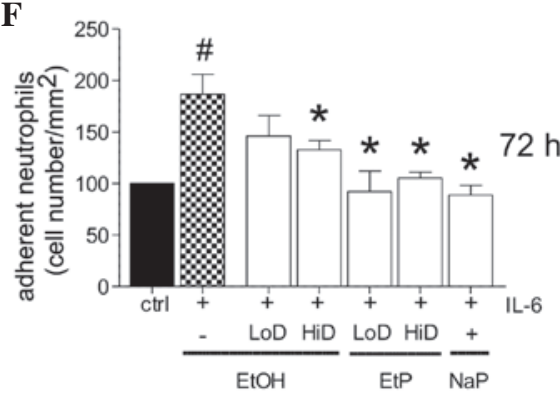

Figure 3. Effects of EtOH, EtP or NaP on the adhesiveness of neutrophils to A549 lung epithelial cells prior to or following IL-6 stimulation (10 ng/ml for 24 h). The cells were treated with EtOH (LoD=85 mM; HiD=170 mM), EtP (LoD=2.5 mM, HiD=10 mM) or NaP (10 mM) 1 h (A) pre- and (B) post-IL-6 stimulation; $24 \mathrm{~h}$ (C) pre- and (D) post-IL-6 stimulation; and $72 \mathrm{~h}$ (E) pre- and (F) post-IL-6 stimulation Following the incubation periods, neutrophils were added and the adhesion capacity after $60 \mathrm{~min}$ was analyzed, expressed as a percentage of the total neutrophils. The data are expressed as the mean \pm standard error of the mean. ${ }^{*} \mathrm{P}<0.05$ vs. untreated IL-6 stimulated control; ${ }^{\#} \mathrm{P}<0.05$ vs. untreated and unstimulated cells. EtOH, ethanol; EtP, ethyl pyruvate; NaP, sodium pyruvate; IL, interleukin; LoD, low dose; HiD, high dose; ctrl, control.

or EtP for different time periods and at different doses prior to or following stimulation of the A549 cells with IL-6. The findings indicated that EtOH and EtP suppressed the IL-6-induced release of IL-8 (Fig. 2). Similar results were observed pre- and post-treatment, which is of significant clinical importance as post-treatment use and effectiveness is likely to be more relevant in the clinical scenario. Similar effects were observed in the adhesion rates of neutrophils to the treated and stimulated epithelial cells (Fig. 3). Post-treatment with either EtOH or EtP was more effective in reducing neutrophil adhesion compared with pre-treatment, possibly due to acute effects on cell surface adhesion molecules (Fig. 3). However, these effects did not correlate with modulations of CD54 in response to IL-6 stimulation, due to inconsistent and rather moderate changes in the expression of CD54 on epithelial cells (Fig. 4), and other adhesion-relevant proteins may be involved.

In numerous models of inflammation, it has been demonstrated that EtOH consumption modulates the inflammatory response, however, scientific disagreement on the exact effects of EtOH on the immune system remains $(1,2,4,6,7)$. While chronic EtOH abuse is associated with unfavorable hyper-inflammatory events, moderate and/or acute intake appears more favorable due to its anti-inflammatory effects in models of inflammation $(4-7,34,35)$. IL-6, a pleiotropic pro-inflammatory cytokine, has been identified as an important contributor to immune regulation and inflammation in various pathologies, including lung injuries in chronic or acute inflammation $(21,36,37)$. IL-6, together with IL-8, accounts to molecules that are involved in the activation, trafficking and infiltration of neutrophils in models of acute lung injury (38). These inflammatory mediators are modulated by EtOH. Acute EtOH use has been demonstrated to reduce the LPS-induced release of IL-6 from macrophages in a dose- and time-dependent manner (39). Furthermore, the anti-inflammatory effects of IL-8 have been reported by Johansson et al, demonstrating that acute treatment of human umbilical vein cells (HUEVCs) with an EtOH dose equal to that used in the present study, and subsequent stimulation of cells with pro-inflammatory mediators, decreased the release of IL-8 (3). In the present study, the release of IL- 8 from lung epithelial cells stimulated with IL-6 was markedly reduced following treatment with either EtOH or EtP (Fig. 2). These anti-inflammatory effects were 

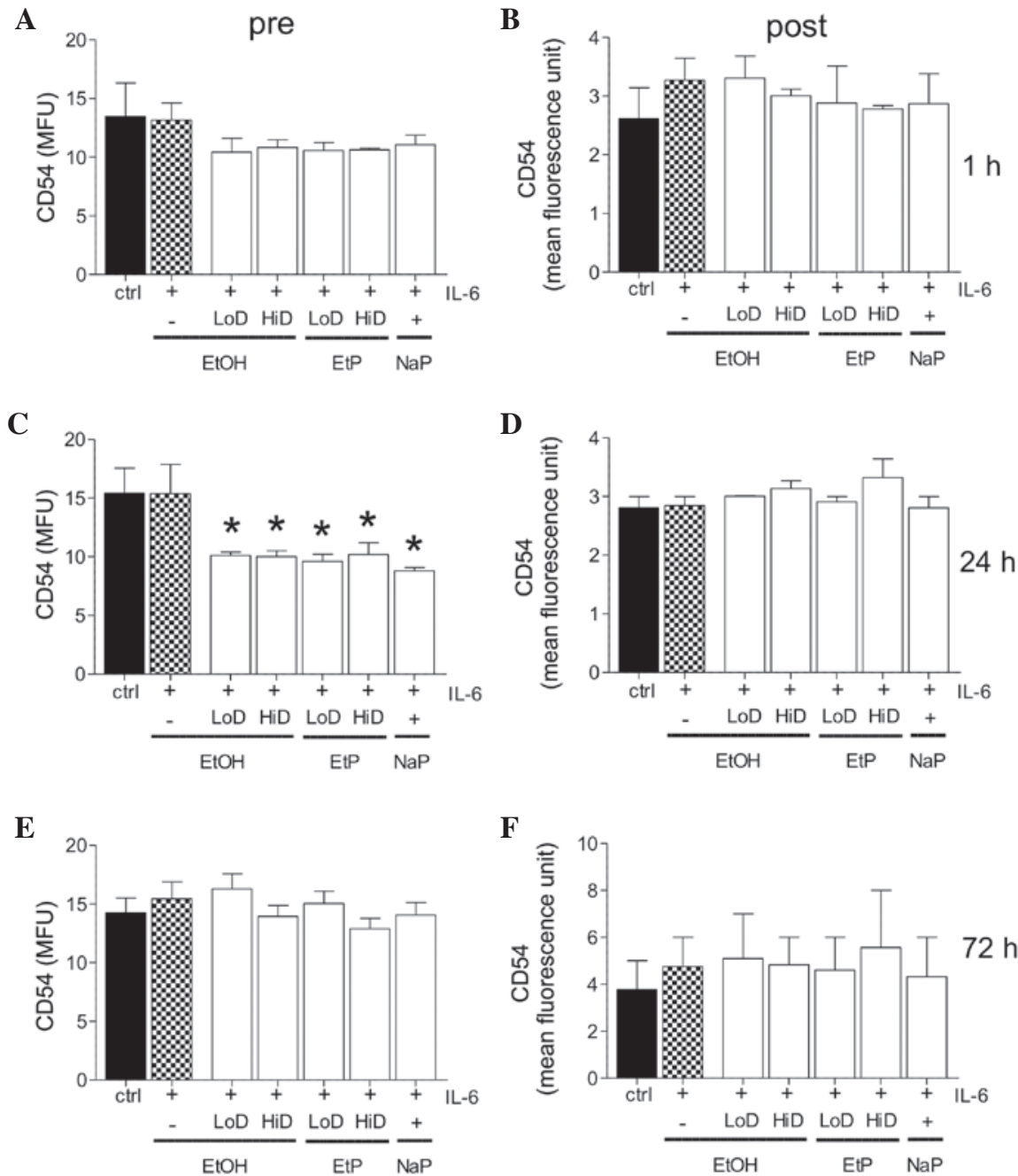

Figure 4. Effects of EtOH, EtP or NaP on the surface expression of CD54 in lung epithelial cells A549 either prior to or following IL-6 stimulation (10 ng/ml for $24 \mathrm{~h})$. The cells were treated with EtOH ( $\mathrm{LoD}=85 \mathrm{mM}, \mathrm{HiD}=170 \mathrm{mM})$, EtP $(\mathrm{LoD}=2.5 \mathrm{mM}, \mathrm{HiD}=10 \mathrm{mM})$ or NaP $(10 \mathrm{mM})$ for $1 \mathrm{~h}$ (A) pre- and (B) post-IL-6 stimulation; $24 \mathrm{~h}(\mathrm{C})$ pre- and (D) post-IL-6 stimulation; and $72 \mathrm{~h}(\mathrm{E})$ pre- and (F) post-IL-6 stimulation. Following incubation, the expression of CD54 was evaluated and expressed as the MFU. The data are presented as the mean \pm standard error of the mean. ${ }^{*} \mathrm{P}<0.05$, vs. untreated IL-6-stimulated control. EtOH, ethanol; EtP, ethyl pyruvate; NaP, sodium pyruvate; CD, cluster of differentiation; IL, interleukin; LoD, low dose; HiD, high dose; ctrl, control; MFU, mean fluorescence unit.

dependent on the incubation duration. Although a short duration of incubation with $\mathrm{EtOH}$ for $1 \mathrm{~h}$ prior to stimulation of the epithelial cells with IL-6 revealed no changes in the release of IL-8, incubation of the cells with a high dose of EtOH decreased the release of IL-8, even when applied following IL-6 stimulation. Longer periods of incubation (24 or $72 \mathrm{~h}$ ) with EtOH prior to or following IL-6 stimulation decreased the release of IL-8 and was independent of the EtOH concentration used (Fig. 2). These findings demonstrated that a high dose of EtOH exerted potent anti-inflammatory effects compared with a low dose. Bhatty et al demonstrated that treating mice with $\sim 87 \mathrm{mM}$ EtOH, and challenging them intraperitoneally with non-pathogenic Escherichia coli, suppressed the production of the majority of known pro-inflammatory cytokines (40). Our previous study revealed that acute EtOH gavage prior to the onset of hemorrhagic shock decreased systemic and local inflammation, including IL-6 levels, in vivo and reduced mortality rates (4). Notably, the anti-inflammatory potential was observed even when EtOH was applied following the inflammatory insult.
The immune response following trauma involves other factors, the hierarchy of which remains to be fully elucidated. Epithelial cells, together with neutrophils, represent the first line of defense in inflammatory conditions, and lung epithelial cells are important in the innate immune response of the lung $(41,42)$. Furthermore, neutrophils are essential components of the host defense and the innate immune system, responsible for combating infections, however, a number of studies have demonstrated that limiting neutrophil presence at sites of inflammation increases organ integrity, as activated neutrophils also have the potential to harm injured tissues (43, 44). The role of neutrophils in the development of lung injury and acute respiratory distress syndrome has been demonstrated in patients recovering from neutropenia and suffering from pneumonia concurrently (45), in which higher mortality rates $(87 \%)$ were observed compared with patients with acute respiratory distress disorder patients, but without preceding neutropenia (52\%) (45). Our previous study demonstrated that the reduced expression of hepatic CD54 following acute EtOH exposure was associated with decreased hepatic neutrophil 
infiltration in an acute model of inflammation (4). Jonsson and Palmblad demonstrated that the increased expression of CD54 in HUVECs following stimulation with LPS increases the adhesion of neutrophils to the endothelial monolayers (30). The present study confirmed that stimulating epithelial cells with IL-6 increased the adhesion of neutrophils, however, the expression of CD54 was not significantly altered (Figs. 3 and 4). Jonsson and Palmblad demonstrated that EtOH inhibited the LPS-induced adhesion of neutrophils to stimulated HUVECs, but did not effect the expression of CD54 (30). The present study demonstrated that EtOH moderately inhibited neutrophil adhesion when it was applied prior to IL-6 stimulation, however, treating the epithelial cells following IL-6 stimulation independently decreased neutrophil adhesion (Fig. 3). The expression of CD54 was not altered by EtOH at any of the incubation periods, with the exception of application of EtOH to the cells prior to IL-6 stimulation, findings that do not correlate with our previous results, but are in line with the those of Jonsson and Palmblad. This suggested that mechanisms other than CD54 exist, by which EtOH modulates PMN-adhesion, possibly via CD31, or CD62L. Therefore, EtOH affected the host immunity in a dose and time-dependent manner, however, support for its clinical application as a therapeutic option is limited. There is a lack of randomized prospective clinical trials on its dose- and time-dependency to support its use; therefore, other treatment options are required. In this regard EtP, a safe and well-tolerated anti-inflammatory drug, may be a realistic approach in the inflammatory settings (22). Comparing the effects of EtOH with EtP in the present study demonstrated that EtP exerted higher anti-inflammatory potential at a lower dose. The present study revealed that EtP was more potent when administered post-treatment and the effects were independent of the incubation duration. This finding is in line with those of Johansson et al, which confirmed the anti-inflammatory potential of EtP (29). Regarding NaP treatment, no consistent data was obtained in the present study. While the effects of NaP were comparable to those of high doses of EtP concerning neutrophil adhesion, it exhibited a weak anti-inflammatory effect. These findings confirmed that the pyruvate moiety of molecules was decisive for adhesion, whereas the ethyl moiety of the EtP and EtOH affected the functional site, based on the adhesion findings.

Taken together, the present study demonstrated a reduction in pro-inflammatory cytokine release from stimulated lung epithelial cells by EtOH and EtP. In addition, the adhesion capacity of neutrophils under inflammatory conditions was decreased by EtOH and by EtP. Furthermore, EtP provided a marked anti-inflamatory and PMN-modulating effects, even when administered post-treatment, and is, therefore, of therapeutical value.

\section{Acknowledgements}

The authors would like to thank Kerstin Kontradowitz, Katrin Jurida and Karam Eldesoqi from the Department of Trauma, Hand and Reconstructive Surgery, University Hospital Frankfurt, Goethe University, for their technical assistance. The present study was supported in part by the German Research Association (grant nos. DFG PE 908/3-1 and RE 3304/5-1).

\section{References}

1. Bird MD, Choudhry MA, Molina PE and Kovacs EJ.: Alcohol and trauma: a summary of the Satellite Symposium at the 30th Annual Meeting of the Shock Society. Alcohol 43: 247-252, 2009.

2. Jurkovich GJ, Rivara FP, Gurney JG, et al: The effect of acute alcohol intoxication and chronic alcohol abuse on outcome from trauma. JAMA 270: 51-56, 1993.

3. Johansson AS, Johansson-Haque K, Okret S and Palmblad $\mathrm{J}$ : Ethyl pyruvate modulates acute inflammatory reactions in human endothelial cells in relation to the NF-kappaB pathway. Br J Pharmacol 154: 1318-1326, 2008.

4. Relja B, Höhn C, Bormann F, et al: Acute alcohol intoxication reduces mortality, inflammatory responses and hepatic injury after haemorrhage and resuscitation in vivo. Br J Pharmacol 165 (4b): 1188-1199, 2012.

5. Cook RT: Alcohol abuse, alcoholism, and damage to the immune system--a review. Alcohol Clin Exp Res 22: 1927-1942, 1998.

6. Mandrekar P, Catalano D, White B and Szabo G: Moderate alcohol intake in humans attenuates monocyte inflammatory responses: inhibition of nuclear regulatory factor kappa $\mathrm{B}$ and induction of interleukin 10. Alcohol Clin Exp Res 30: 135-139, 2006.

7. Szabo G, Mandrekar P, Girouard L and Catalano D: Regulation of human monocyte functions by acute ethanol treatment: decreased tumor necrosis factor-alpha, interleukin-1 beta and elevated interleukin-10, and transforming growth factor-beta production. Alcohol Clin Exp Res 20: 900-907, 1996.

8. Relja B, Omid N, Kontradowitz K, et al: Decreased inflammatory responses of human lung epithelial cells after ethanol exposure are mimicked by ethyl pyruvate. Mediators Inflamm 2014: 781519. 2014.

9. Li G, Keyl PM, Smith GS and Baker SP: Alcohol and injury severity: reappraisal of the continuing controversy. J Trauma 42: $562-569,1997$.

10. Rehm J, Room R, Graham K, Monteiro M, Gmel G and Sempos CT: The relationship of average volume of alcohol consumption and patterns of drinking to burden of disease: an overview. Addiction 98: 1209-1228, 2003.

11. Ruiz M, Ewig S, Torres A, et al: Severe community-acquired pneumonia. Risk factors and follow-up epidemiology. Am J Respir Crit Care Med 160: 923-929, 1999.

12. Bagby GJ, Zhang P, Stoltz DA and Nelson S: Suppression of the granulocyte colony-stimulating factor response to Escherichia coli challenge by alcohol intoxication. Alcohol Clin Exp Res 22: 1740-1745, 1998.

13. Boé DM, Nelson S, Zhang P and Bagby GJ: Acute ethanol intoxication suppresses lung chemokine production following infection with Streptococcus pneumoniae. J Infect Dis 184: 1134-1142, 2001

14. Boé DM, Nelson S, Zhang P, Quinton L and Bagby GJ: Alcohol-induced suppression of lung chemokine production and the host defense response to Streptococcus pneumoniae. Alcohol Clin Exp Res 27: 1838-1845, 2003.

15. Faunce DE, Gregory MS and Kovacs EJ: Effects of acute ethanol exposure on cellular immune responses in a murine model of thermal injury. J Leukoc Biol 62: 733-740, 1997

16. Messingham KA, Faunce DE and Kovacs EJ. Alcohol, injury, and cellular immunity. Alcohol 28: 137-149, 2002

17. Zhang P, Bagby GJ, Boé DM, et al: Acute alcohol intoxication suppresses the CXC chemokine response during endotoxemia. Alcohol Clin Exp Res 26: 65-73, 2002.

18. Berger K, Ajani UA, Kase CS, et al: Light-to-moderate alcohol consumption and risk of stroke among U.S. male physicians. N Engl J Med 341: 1557-1564, 1999.

19. Lustenberger T, Inaba K, Barmparas G, et al: Ethanol intoxication is associated with a lower incidence of admission coagulopathy in severe traumatic brain injury patients. J Neurotrauma 28: 1699-1706, 2011.

20. Sesso HD: Alcohol and cardiovascular health: recent findings. Am J Cardiovasc Drugs 1: 167-172, 2001.

21. Rubini A: Interleukin-6 and lung inflammation: Evidences of a causing role in inducing respiratory system resistance increments. Inflamm Allergy Drug Targets 12: 315-321, 2013.

22. Fink MP: Ethyl pyruvate. Curr Opin Anaesthesiol 21: 160-167, 2008.

23. Kao KK and Fink MP: The biochemical basis for the anti-inflammatory and cytoprotective actions of ethyl pyruvate and related compounds. Biochem Pharmacol 80: 151-159, 2010. 
24. Wang Y, Yin P, Huang S, Wang J and Sun R: Ethyl pyruvate protects against lipopolysaccharide-induced white matter injury in the developing rat brain. Int J Dev Neurosci 31: $181-188,2013$.

25. Cai B, Brunner M, Wang H, Wang P, Deitch EA and Ulloa L: Ethyl pyruvate improves survival in awake hemorrhage. J Mol Med-Berl 87: 423-433, 2009.

26. Jacobs CC, Holcombe SJ, Cook VL, Gandy JC, Hauptman JG and Sordillo LM: Ethyl pyruvate diminishes the inflammatory response to lipopolysaccharide infusion in horses. Equine Vet J 45: 333-339, 2013

27. Li LF, Kao KC, Yang CT, Huang CC and Liu YY: Ethyl pyruvate reduces ventilation-induced neutrophil infiltration and oxidative stress. Exp Biol Med (Maywood) 237: 720-727, 2012.

28. Holzer K, Schübel F, Konietzny P, Wilhelm K, Bechstein WO and Henrich D: Interleukin 8 mRNA gene expression in peripheral and intra-abdominal neutrophils during human secondary peritonitis. Shock 23: 501-506, 2005.

29. Johansson AS and Palmblad J: Ethyl pyruvate modulates adhesive and secretory reactions in human lung epithelial cells. Life Sci 84: 805-809, 2009.

30. Jonsson AS and Palmblad JE: Effects of ethanol on NF-kappaB activation, production of myeloid growth factors, and adhesive events in human endothelial cells. J Infect Dis 184: 761-769, 2001

31. Maiya R, Buck KJ, Harris RA and Mayfield RD: Ethanol-sensitive sites on the human dopamine transporter. J Biol Chem 277: 30724-30729, 2002

32. Famili A, Ammar DA and Kahook MY: Ethyl pyruvate treatment mitigates oxidative stress damage in cultured trabecular meshwork cells. Mol Vis 19: 1304-1309, 2013.

33. Zhang X, Yin P, Di D, et al: IL-6 regulates MMP-10 expression via JAK2/STAT3 signaling pathway in a human lung adenocarcinoma cell line. Anticancer Res 29: 4497-4501, 2009.

34. Ajisaka $\mathrm{H}$, Okajima M, Goto $\mathrm{Y}$, Taniguchi $\mathrm{T}$ and Inaba $\mathrm{H}$ Effects of acute low-dose ethanol on inflammatory reactions to endotoxin-induced shock in rats. J Toxicol Sci 37: 649-654, 2012.
35. Hubeau C, Kubera JE, Masek-Hammerman K and Williams CM: Interleukin-6 neutralization alleviates pulmonary inflammation in mice exposed to cigarette smoke and poly(I:C). Clin Sci (Lond) 125: 483-493, 2013.

36. Relja B, Henrich D, Wetzel G, et al: Effects of acute ethanol gavage on intestinal integrity after hemorrhage/resuscitation. Scand J Gastroenterol 48: 448-458, 2013.

37. Smolen JS, Schoels MM, Nishimoto N, et al: Consensus statement on blocking the effects of interleukin- 6 and in particular by interleukin-6 receptor inhibition in rheumatoid arthritis and other inflammatory conditions. Ann Rheum Dis 72: 482-492. 2013.

38. Reiss, LK, Uhlig, U and Uhlig, S: Models and mechanisms of acute lung injury caused by direct insults. Eur J Cell Biol 91: 590-601, 2012.

39. Karavitis J, Murdoch EL, Gomez CR, Ramirez L and Kovacs EJ Acute ethanol exposure attenuates pattern recognition receptor activated macrophage functions. J Interferon Cytokine Res 28: 413-422, 2008

40. Bhatty M, Jan BL, Tan W, Pruett SB and Nanduri B: Role of acute ethanol exposure and TLR4 in early events of sepsis in a mouse model. Alcohol 45: 795-803, 2011.

41. Bals R and Hiemstra PS. Innate immunity in the lung: how epithelial cells fight against respiratory pathogens. Eur Respir J 23: 327-333, 2004

42. Fogli LK, Sundrud MS, Goel S, et al: T cell-derived IL-17 mediates epithelial changes in the airway and drives pulmonary neutrophilia. J Immunol 191: 3100-3111, 2013.

43. Segel GB, Halterman MW and Lichtman MA: The paradox of the neutrophil's role in tissue injury. J Leukoc Biol 89: 359-372, 2011.

44. Weckbach S, Hohmann C, Braumueller, et al: Inflammatory and apoptotic alterations in serum and injured tissue after experimental polytrauma in mice: Distinct early response compared with single trauma or "double-hit" injury. J Trauma Acute Care Surg 74: 489-498, 2013.

45. Rhee CK, Kang JY, Kim YH, et al: Risk factors for acute respiratory distress syndrome during neutropenia recovery in patients with hematologic malignancies. Crit Care 13: R173, 2009. 